\title{
Treatment of HCV-Related Mixed Cryoglobulinemia
}

\author{
Dilia Giuggioli ${ }^{1}$, Marco Sebastiani ${ }^{1}$, Michele Colaci ${ }^{1}$, Poupak Fallahi ${ }^{2}$, Laura Gragnani ${ }^{3}$, \\ Anna Linda Zignego ${ }^{3}$, Alessandro Antonelli ${ }^{2}$ and Clodoveo Ferri ${ }^{1, *}$
}

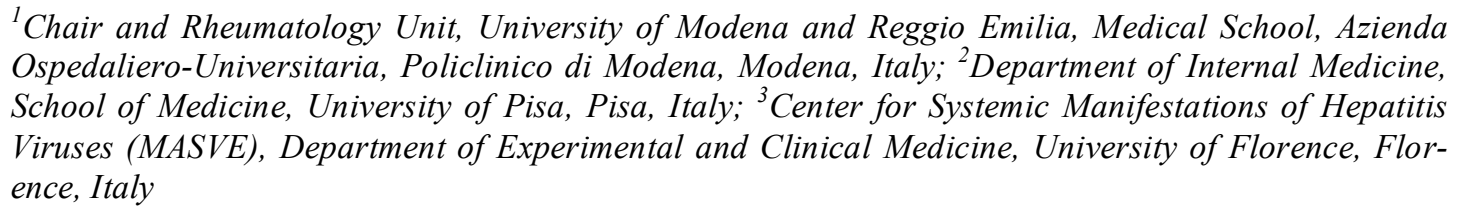

Abstract: Mixed cryoglobulinemia syndrome (MCs) is a systemic vasculitis, involving skin, joints, peripheral nerves, and several internal organs. Hepatitis $\mathrm{C}$ virus (HCV) is recognized as the etiologic agent for the majority of MCs patients, as well as of number of autoimmune, lymphoproliferative, and neoplastic disorders. In this context, HCV-related MCs represents an important model autoim-

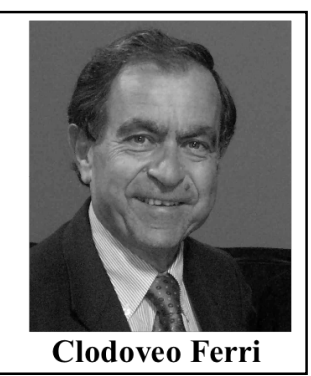
mune/neoplastic disease triggered by a virus in humans. With regard the therapeutic strategies of MCs, we can treat these patients at different steps by means of etiological (antivirals), pathogenetic, symptomatic drugs (mainly immunosuppressors, corticosteroids, plasmapheresis). In the majority of individuals, MCs shows a mild, slow-progressive clinical course needing only symptomatic treatments, generally low doses of corticosteroids. Considering the etiopathogenesis of MCs, the eradication of HCV should be considered the gold standard in the treatment of MCs. The use of combined peginterferon- $\alpha$ /ribavirin and/or novel antiviral drugs may lead to HCV eradication in a significant percentage of cases with possible remission of MCs. On the other hand, the presence of rapidly progressive, diffuse vasculitis with multiple organ involvement may be successfully treated with aggressive immunosuppressive and anti-inflammatory therapies, mainly based on cyclophosphamide or rituximab, high dose corticosteroids, and plasma aphaeresis. Moreover, sequential/combined antiviral or immunosuppressive treatments could represent an useful therapeutic strategy particularly in MCs patients with major clinical manifestations.

The treatment of MCs should be decided for every patient according to the severity of clinical picture. Thus, a careful follow-up of the disease is necessary, with particular attention to the possibility of cancers onset, such as B-cell lymphoma. The present review focuses on the different therapeutic strategies in patients with MCs, including the treatment of cryoglobulinemic skin ulcers, which represents one of the most discouraging complications of the disease.

Keywords: Hepatitis C virus, mixed cryoglobulinemia, cryoglobulinemic vasculitis, lymphoma, skin ulcers, rituximab.

\section{INTRODUCTION}

Cryoglobulinemia refers to a laboratory abnormality characterized by the presence in the serum of one or more immunoglobulins, which precipitate at temperatures $<37^{\circ} \mathrm{C}$ and re-dissolve on re-warming $[1,2]$. It is classified into 3 types according to Ig composition [3]: namely, type I cryoglobulins are single monoclonal immunoglobulins related to hematological disorders, often B-cell lymphoproliferation, and it is per se frequently asymptomatic. Mixed cryoglobulinemia type II $(\mathrm{IgG}+$ monoclonal IgM) or type III $(\operatorname{IgG}+$ polyclonal IgM) consists of polyclonal IgG with or without monoclonal IgM, respectively, which has rheumatoid factor activity [1-3].

Cryoglobulins may be commonly detected in several infectious or autoimmune or neoplastic disorders [2-5]. By

\footnotetext{
*Address correspondence to this author at the Rheumatology Unit, Dpt of Internal Medicine, University of Modena and Reggio E., Policlinico di Modena, Via del Pozzo, 71, 41100 Modena, Italy; Tel: +39-059-4222279; Fax: +39-059-4223007; E-mail: clferri@unimore.it
}

contrast, mixed cryoglobulinemia syndrome (MCs) is regarded as distinct disorder, which can be classified among systemic vasculitides, in the subgroup involving smallmedium sized vessels; MCs and cryoglobulinemic vasculitis (CV) are synonyms [5]. The histological hallmark of the disease is the leukocytoclastic vasculitis secondary to the vascular deposition of circulating cryo- and non- cryoprecipitable immune-complexes (IC) and complement [5]. The vasculitic lesions are responsible for different MCs clinical features, including skin and internal organ involvement [1-3, 5]. The prevalence of MCs is geographically heterogeneous; the disease is less common in Northern Europe or Northern America than in Southern Europe [5, 6]. It is considered a rare disorder, even though there are no adequate epidemiological studies regarding its world prevalence, up to date. MCs is characterized by a clinical polymorphism, thus patients with vasculitis are often referred to different specialty centres, and a correct diagnosis might be delayed or overlooked entirely; for the same reasons, the true prevalence of MCs might be underestimated [2,5]. Overall, MCs is more common in females than in males (F/M 3:1), while the dis- 
ease onset is particularly common in the fourth-fifth decades and in older people, and rarely seen in the young people. From a clinico-pathogenetic point of view MCs could represents a crossroad between classical rheumatic/autoimmune diseases, such as rheumatoid arthritis, Sjögren's syndrome, and lymphoproliferative/neoplastic disorders $[2,5]$.

\section{ETIOPATHOGENESIS OF MCS}

A causative role of hepatitis $\mathrm{C}$ virus (HCV) in the large majority of patients has been clearly ascertained on the basis of epidemiological, pathological, and laboratory studies [7$11]$.

The prevalence of anti-HCV antibodies and/or HCVRNA in subjects with MCs varies from $70 \%$ to $>90 \%$ among different patients' populations [12, 13]; this finding relegates the term 'essential' MCs to a few patients [14]. The clinical development of MCs is closely related to the natural history of chronic HCV infection; generally, this immunological disorder represents a late complication of viral infection. The appearance of different MCs phenotypes may be the result of genetic and environmental cofactors, which remain poorly known $[4,5]$. The etiopathogenetic cascade of MCs and other disorders related to HCV is summarized in Fig. (1) (left). The production of different organ-specific and systemic disorders is presumably a multi-factorial and multistep process: the trigger factors include some infectious agents, mainly HCV, host genetic predisposition, and eventual unknown environmental/toxic causes. Some HCV antigens, i.e. HCV core, envelop E2, NS3, NS4, NS5A proteins, may represent a chronic stimulus to lymphocytes through specific receptors, such as CD81 that may bind with the viral protein E2 [15-17]. Specific HLA alleles may represent predisposing factors [18], as well as the metabolic and the hormonal status of infected individuals. The result is a "benign" B-cell proliferation with autoantibody overproduction, among which the rheumatoid factor (RF), and the cryoprecipitable and non-cryoprecipitable immune- complexes (IC) [19]. These immunological abnormalities may be correlated with different specific autoimmune disorders, including the systemic manifestations of MCs. On the other side, the activation of $\mathrm{Bcl} 2$ proto-oncogene, which is responsible for prolonged survival of B- lymphocytes, may be a precondition to other genetic aberrations, which ultimately may produce overt B-cell lymphomas and other malignant neoplasias [20, 21]. The appearance of different malignancies may be observed in a small but not negligible percentage of patients, generally late manifestation of chronic $\mathrm{HCV}$ infection with/without MCs [20, 22-25]. Both immune-mediated and cancer diseases show clinical, serological and pathological overlaps. More frequently, autoimmune organ-specific manifestations may

precede the development of systemic conditions, which in same individuals may be complicated by malignancies (Fig. 1 left). From the other side, it is possible to observe the appearance of some autoimmune manifestations in patients with malignancies [23]. On the whole, the proposed ' $\mathrm{HCV}$ syndrome' encompasses this multiform complex of HCVrelated clinical manifestations, among which the MCs represents a crossing road between autoimmune and neoplastic disorders [26].

\section{CLINICAL FEATURES}

The MCs is characterized mainly by the classical triad purpura, weakness, arthralgias; furthermore, clinical picture encompasses chronic hepatitis, membrano-proliferative glomerulonephritis (MPGN), peripheral neuropathy, skin ulcers, and, less frequently, lymphatic and hepatic cancers [5; Fig. 2]. The presenting symptoms are largely variable among MCs patients. At the initial observation, patients show different clinical and serological patterns, ranging from apparently isolated presence of mixed cryoglobulins in the serum to the complete cryoglobulinemic syndrome (Fig. 2). For a correct clinical classification we can regard the MCs as a combination of serological parameters (mixed cryoglobulins, RF, low C4) and clinical or pathological features, (purpura and leukocytoclastic vasculitis, respectively) [27]. While, cryoglobulins may be more frequently found in asymptomatic patients with chronic HCV infection [5]. Fig. (2) shows the main clinical features of MCs. Cutaneous manifestations represent the most frequent features of the MCs [2, 5]; the hallmark is the orthostatic, generally intermittent purpura, ranging from sporadic petechiae to severe vasculitic lesions, while chronic ulcers of legs and feet may affect a significant percentage of patients (Fig. 3). MC patients may frequently develop a permanent ochreous coloration on the legs due to repeated poussèes of purpura (Fig. 3). Skin manifestations of the legs are the direct consequence of vasculitis, with the eventual coexistence of chronic venous insufficiency, physical stress, such as prolonged standing or muggy weather. The majority of MCs patients suffers from arthralgias, while synovitis, mild and non-erosive oligoarthritis if present, is quite rare [28, 29, Sebastiani et al. in the present issue]. Peripheral neuropathy is a typical complication of MCs, frequently as mild sensory neuritis in about $80 \%$ of MCs patients [30, 31]; it is clinically characterized by paresthesias, pain or burning sensation in the lower limbs, often with night-time exacerbation. Therefore, the patients' quality of life is often compromised, given the chronicity of these symptoms and their scarce response to treatments. Less frequently, peripheral neuropathy may be complicated by severe asymmetrical motor manifestations; in some subjects it may be associated with the alpha-interferon treatment [32] system involvement, are rarely described [5], while it is often difficult to discriminate these features from the effect of possible concomitant atherosclerotic disease. Moreover, mild-tomoderate chronic hepatitis can be observed at any time during the long-lasting follow-up as expression of the underlying HCV infection. Chronic hepatitis may evolve to cirrhosis, and less frequently may be complicated by hepatocellular carcinoma [23, 33]. With regards the overall prognostic value of hepatic manifestations, the liver involvement in patients with MCs seems to be less severe in comparison to chronic HCV hepatitis alone [2, 5].

Xerophthalmia and xerostomia are present in almost half MCs patients; yet, only a few cases meet the classification criteria for primary Sjögren's syndrome [34, 35]. Raynaud's phenomenon is reported in about $25 \%$ of patients, as showed in Fig. (2). MPGN type 1 is an important organ involvement that could severely affect the prognosis and the survival of MCs patients [36, 37]. MC-related MPGN is a typical 


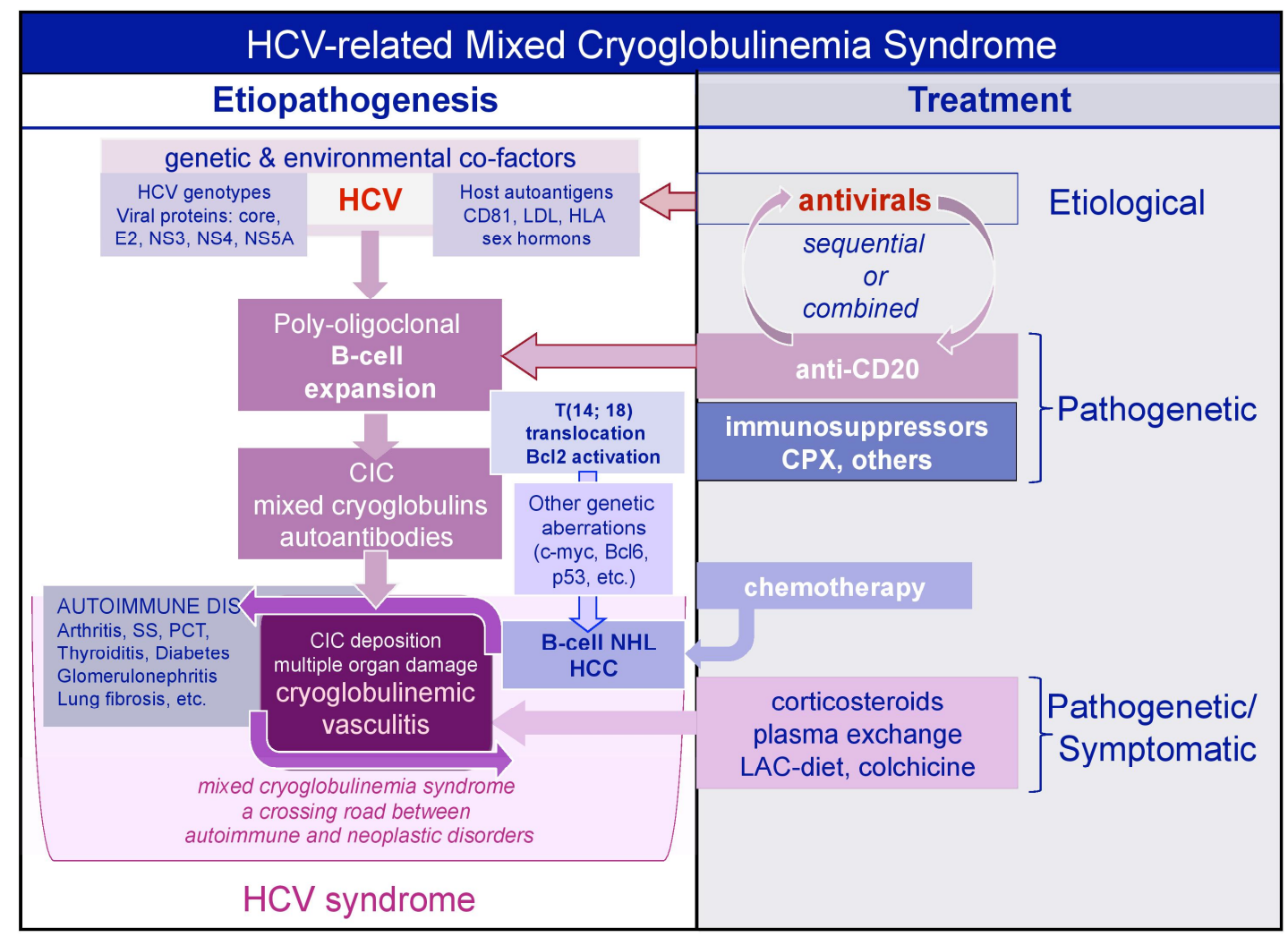

Fig. (1). Left: etiopathogenetic cascade of MCs. It is a multi-factorial and multi-step process, including HCV, predisposing host factors, and unknown environmental/toxic triggers. The main consequence is a "benign" B-cells proliferation with a variety of autoantibody production, among which RF, and cryo- and non-cryoprecipitable immune-complexes, which may be correlated to various autoimmune disorders, including the MCs. Moreover, the activation of Bcl2 proto-oncogene, followed by other genetic errors, may lead to frank B-cell lymphomas and other malignancies. Both immunological and neoplastic disorders show overlapping clinical, serological and pathological pictures. In several cases, autoimmune organ-specific disorders may evolve to systemic conditions, and less frequently to malignancies. On the other hand, it is not rare that patients with cancers may develop autoimmune manifestations. In this context, cryoglobulinemic vasculitis represents a crossing road between autoimmunity and neoplastic disorders. While the overall symptom complex triggered by viral infection may be termed ' $\mathrm{HCV}$ syndrome'.

glomerulonephritis mediated by IC deposition, although other immunological mechanisms have also been hypothesized. A widespread vasculitis of medium-small sized vessels may be sparked off in a small subset of patients and may involve skin, kidney, lungs, central nervous system, and gut $[2,5,38]$. Interstitial lung involvement (mainly subclinical alveolitis) has been anecdotally described in MCs, as well as in $\mathrm{HCV}$ patients without cryoglobulinemia [2, 39]. From a practical point of view, there is no relationship between the severity or the activity of clinical pictures, and the levels of cryoglobulins or complement in the serum [5]. Some endocrinological disorders may be observed in MCs, mainly autoimmune thyroiditis with subclinical hypothyroidism, thyroid cancer, and an increased incidence of diabetes mellitus type 2 [40-43]. B-cell lymphoma is the most frequent malignancy complicating MCs [24]. It may be related to the peripheral B- cells expansion and 'indolent' lymphoid infiltrates localized in the liver and bone marrow of MCs patients [25].

Other neoplasms, such as the hepatocellular carcinoma or the papillary thyroid cancer, are less frequently reported [33, 42]. In this light, the MCs can be considered as a preneoplastic condition; therefore, a careful follow-up is mandatory, even in the presence of mild MCs. Low complement activity, with the typical pattern of low/undetectable $\mathrm{C} 4$ and normal $\mathrm{C} 3$, is almost regularly detectable in $\mathrm{MCs}$, independently from the disease activity (Fig. 2).

Interestingly, a sudden increase of $\mathrm{C} 4$ to unusual high levels can be observed in MCs patients developing B-cell lymphoma [5].

\section{TREATMENT}

MCs is characterized by mild, slow progressive clinical course, but in a significant number of patients with moderate/severe cutaneous and/or visceral organ involvement the therapeutical approach may result particularly challenging, considering the multifaceted etiopathogenesis and the clinical polymorphism of the disease.

A correct strategy should deal with 3 concomitant factors: HCV infection, autoimmune disorder, and pre neoplastic alteration (Fig. 1). Fig. (1) shows the main steps of the etiopathogenetic process, i.e. viral infection, Blymphocyte proliferation, and cryoglobulinemic vasculitis (Fig. 1); accordingly, we may treat MCs patients at these 3 levels by means of etiologic, pathogenetic, and/or symptomatic therapies $[2,5,26]$. Considering the $\mathrm{HCV}$ as triggering 


\section{Mixed Cryoglobulinemia Syndrome}

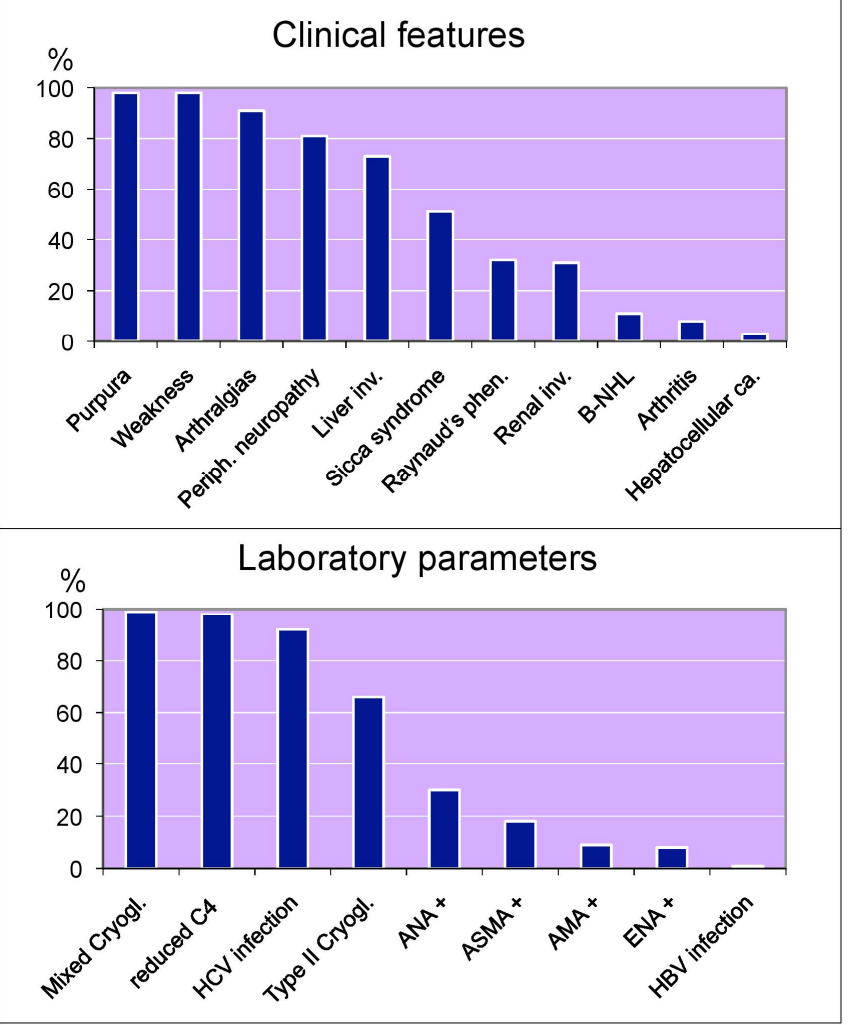

Fig. (2). Clinico-serological features of MCs.

HCV: hepatitis C virus; HBV: hepatitis B virus; ANA: antinuclear antibodies: ENA: anti-extractable nuclear antigen antibodies; ASMA: anti-smooth muscle antibodies AMA: antimitochondrial antibodies; B-NHL: B-cell non- Hodgkin's lymphoma

and possibly perpetuating agent of the MCs through a chronic trigger on the immune system, HCV eradication treatment should be attempted in all patients with HCVassociated MCs [5, 44, 45; see also Zignego et al. in the present issue]. Initially, the treatment with Interferon alpha $(\mathrm{IFN} \alpha)$ was associated with a relatively poor response and a high rate of disease relapses [46]. Combination therapy with IFN $\alpha$ plus ribavirin showed a greater antiviral efficacy with sustained virological response in a variable percentage of patients with chronic hepatitis $\mathrm{C}$, as well as much better short- and long-term results in MCs patients than reported with IFN $\alpha$ monotherapy $[46,47]$. Indeed, the beneficial effect reached with IFN $\alpha$ and ribarivin is often transient or associated with important immune-mediated side effects, such as peripheral neuropathy, thyroiditis, and rheumatoidlike arthritis [48]. It is possible that IFN $\alpha$, both antiviral and immunomodulating agent, can trigger or exacerbate some symptoms in predisposed subjects; therefore, IFN $\alpha$ should be avoided at least in patients with peripheral neuropathy. The efficacy of antiviral therapy progressively increased during the time with the introduction of Peg-IFN and RBV, and ultimately it became very promising with the novel combination therapies, including interferon-free drugs [49; see also Zignego et al. in the present issue]. The long-term effects of $\mathrm{HCV}$ eradication needs to be deeply investigated; particularly the outcome of HCV-related immunological alterations, including cryoglobulinemia and its clinical manifestations. Although anecdotal or limited to small patients' series, preliminary observations suggest that the effects of HCV eradication on MCs remain quite unpredictable [50]. One of the possible factors could be the disease duration at the time of HCV eradication with self-perpetuating autoimmune mechanism underlying long-lasting MCs; therefore, it is possible to hypothesize a point of no return in the natural history of MCs. In the setting of HCV eradication, a recent study suggested that the presence of MCs may represent a negative prognostic factor of virological response, while the clearance of HCV may lead to persistent resolution or improvement of MCs [50]. The more recent use of triple therapy with PegIFN, ribavirin, and a specifically targeted antiviral agent so called DAAs (i.e. Boceprevir, Telaprevir, Sofosbuvir) led to improve sustained virological response rates in patients infected by HCV genotype 1 [51]. Boceprevir-based therapy was reported as safe and effective in cryoglobulinemic patients [50; see also Zignego et al. in the present issue], while the use of sofosbovir produced still contrasting effects [52]. Further studies are needed to confirm and clarify the activity of these IFN-free antiviral combination in larger series of MCs patients. Hopefully, a vaccination [53] with recombinant $\mathrm{HCV}$ proteins in $\mathrm{HCV}$-infected patients could prevent the progression of viral infection and possibly stop the virusdriven autoimmune disease. Immunosuppression with rituximab or cyclophosphamide represents the pathogenetic treatment of patients with MCs (Fig. 1 right and 4). These treatments include also steroids, low antigen content (LAC) diet, and plasma exchange $[2,26,46,54,55]$. The immunosuppressors are typically reserved for patients with severe disease features, such as MPGN, sensory-motor neuropathy and life- threatening complications. In these patients high dose corticosteroids and immunosuppressors has been used for the control of severe vasculitis lesions, alone or as sequential/combined therapy, while awaiting the generally slow response to antiviral treatments [46, 54-56]. Low dose of steroids may help to control minor intermittent inflammatory symptoms, such as sporadic purpura, arthralgia, weakness, mild peripheral sensory neuropathy $[2,13,26]$. Plasmapheresis represents a pathogenetic/symptomatic therapy by removing circulating IC, mainly cryoglobulins, complement, and other pro-inflammatory agents; it is particularly effective for rapidly progressive glomerulonephritis, sensory-motor neuropathy, diffuse vasculitis, and/or severe skin ulcers $[2,57]$.

Both traditional plasma aphaeresis and double-filtration plasma exchange, in combination with steroids and immunosuppressors may be able to markedly reduce the circulating immune- complex levels with rapid symptom amelioration [2]. Immunosuppressors (rituximab or cyclophosphamide) may reinforce the beneficial effect of plasma exchange and also prevent the possible rebound phenomenon during the tapering of aphaeretic sessions [38, 46]. LAC-diet can improve the clearance of circulating $\mathrm{IC}$ by restoring the activity of the reticule-endothelial system, overloaded by large amounts of circulating cryoglobulins [58]. This particular dietetic treatment is able to reduce the input of macromolecules derived from food crossing the mucosal barrier of the gut; some foods, particularly dairy products and eggs, have a potential antigenic activity, thus might be involved in the 


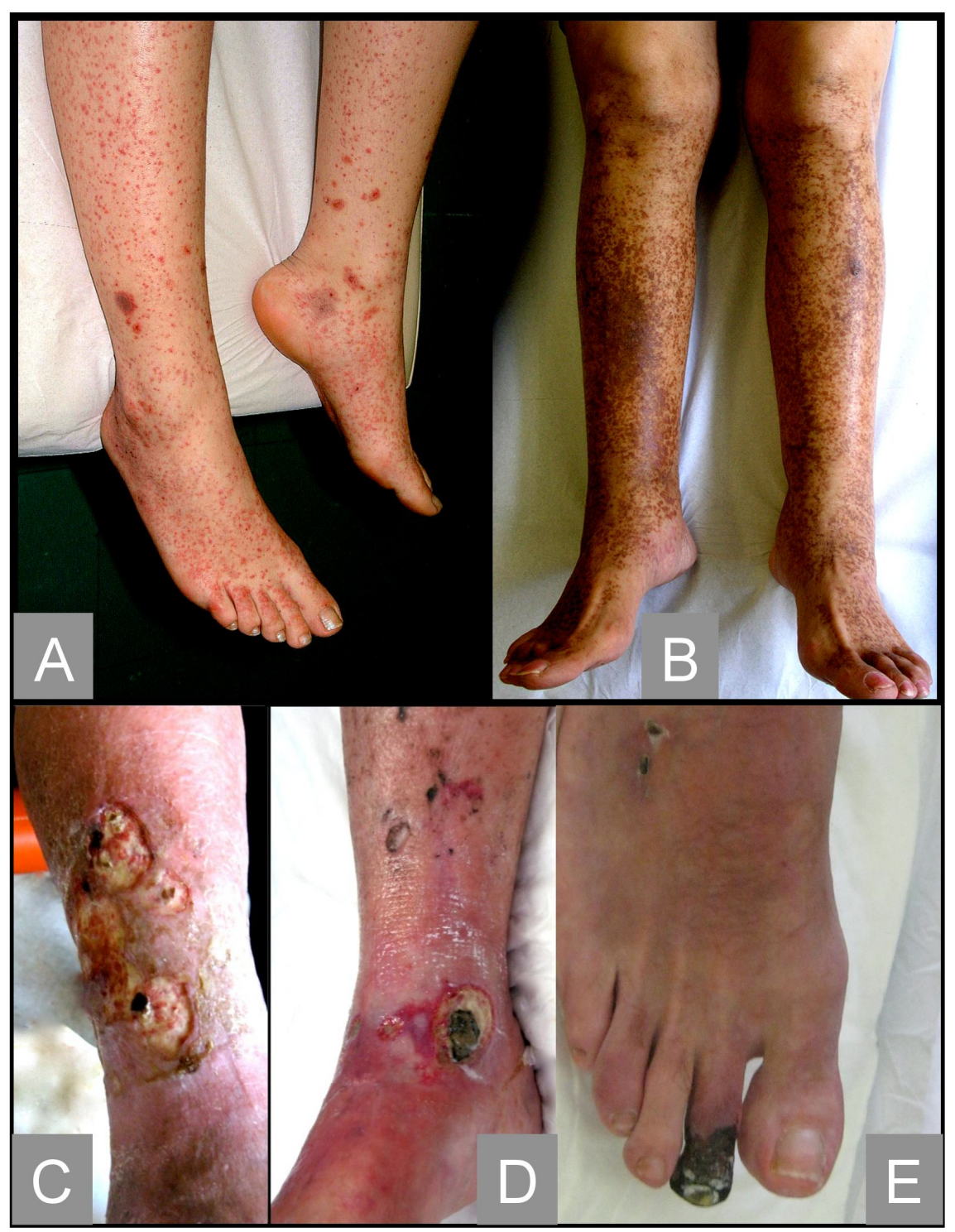

Fig. (3). Cutaneous manifestations of MCs. different skin manifestations of cryoglobulinemic vasculitis: a) orthostatic purpura secondary to necrotizing leukocytoclastic vasculitis of the skin; b) symmetrical hyperpigmentation of the skin on the legs after several poussèes of purpura; the latter and these permanent ochraceous lesions represent the typical skin manifestations of CV; $\mathrm{c}-\mathrm{d}$ ) more severe vasculitic manifestations showing multiple skin ulcers with the presence of central necrotic tissue and slough (adherent fibrous material derived from proteins, fibrin and fibrinogen) in the wound bed. The presence of devitalized tissue acts as a physical barrier to epidermal cell migration and healing process; the borders of skin ulcers are irregular and scarcely reactive. e) distal gangrene of the second toe of the right foot.

pathogenesis of some immune-mediated disorders. The reduction of the alimentary input of macromolecules directed to mononuclear phagocyte system may improve its function in those diseases characterized by abnormal endogenous production of IC, i.e. mixed cryoglobulinemia. In patients with clinically mild MCs symptoms, LAC-diet may be usefully employed along with low dose of steroids (prednisone 2-7.5 mg/day), often sufficient to control mild MCs manifestations (arthralgias, sporadic purpura) [2, 54].

Anti-CD20 monoclonal antibody (rituximab, RTX) represents the first-line immunosuppressive/immunomodulating treatment of $\mathrm{HCV}$-related MCs during the last years considering its efficacy and safety $[49,55,56,59]$. In the first reports, the main indication for RTX was the failure or intoler- ance to other treatments, and/or associated lymphoma [60, 61]. RTX may be usefully employed alone or in combination/sequence with Peg- IFN-ribavirin; in MCs patients, combined antiviral (boceprevir) and RTX treatment may be followed by complete remission of MCs manifestations and sustained virological response (see also Zignego et al. in this issue). Tailoring specific treatment strategies In clinical practice, the treatment of MCs should be tailored for every patient, according to the severity of clinical picture (Fig. 4). Clinically asymptomatic subjects usually do not need any therapy, even in presence of high levels of cryoglobulins. However, antiviral therapy might represent the etiological treatment also for patients with mild MCs manifestations, with the purpose to avoid the progression of the immunological disorder as well as of the most severe hepatic 


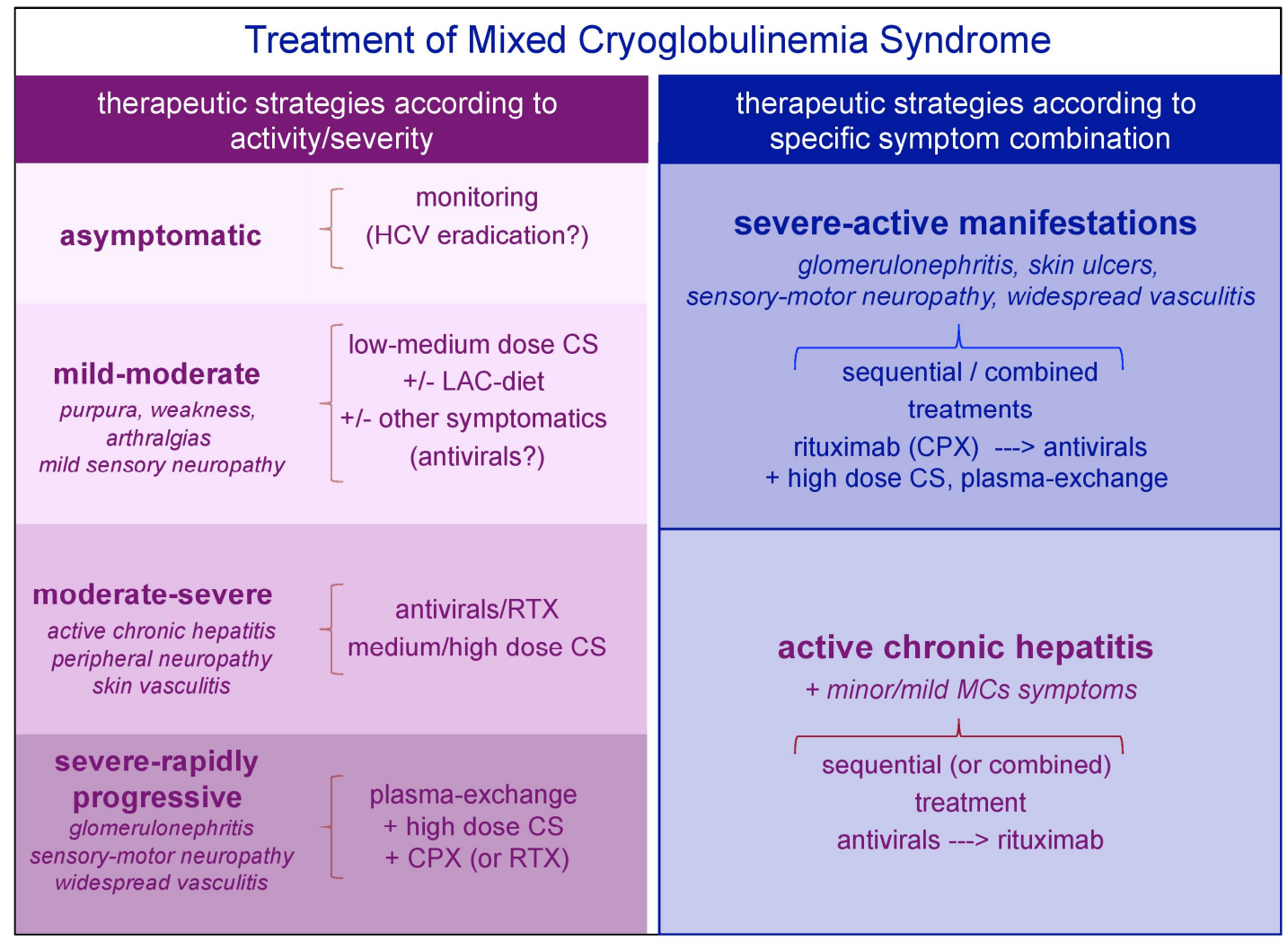

Fig. (4). The treatment of MCs may be modulated according to the clinical status of patients (see text). CPX, cyclophosphamide; CS, corticosteroid; LAC, low antigen-content; RTX, rituximab.

manifestations. Patients with mild to moderate MCs (i.e. arthralgia, purpura, and/or mild sensory polyneuropathy) may be treated with low dose corticosteroids and/or LACdiet able to control these minor inflammatory manifestations. On the contrary, sequential/combined antiviral and immunosuppressive therapy should be considered in patients with moderate-severe symptoms; an attempt with antivirals might be firstly used in subjects with active chronic hepatitis, with high risk to develop cirrhosis and/or hepatocellular carcinoma. Finally, patients with severe vasculitic manifestations, MPGN, sensory-motor neuropathy, and/or widespread vasculitis must be timely treated with high doses of steroids, plasma exchange, and/or cyclophosphamide or RTX. For the above manifestations the therapeutic strategy should take into account the possible comorbidities (Fig. 4, right). For MCs patients presenting with the fulminant clinical variants, including peripheral gangrene, rapidly progressive glomerulonephritis, abdominal, cardiac, pulmonary, central nervous system vasculitic involvements and/or the rare hyperviscosity syndrome, plasmapheresis may lead to immediate beneficial effects. In general, the apheresis should be followed by immunosuppression, in order to avoid post- apheretic rebound of symptoms. Overall, a careful monitoring of MCs patients is mandatory, with particular attention to metabolic, autoimmune, and neoplastic complications [62-66]. The treatment of MCs associated with malignant lymphoproliferation, mainly B-cell NHL, is a priority than of the underlying hematological disorder. Treatment of cryoglobulinemic skin ulcers The updated review of the literature revealed the presence of skin ulcers in around a quarter of MCs patients [5]. These are often non-healing cutaneous lesions, possibly complicated by local infection and gangrene; moreover, they severely affect the patients' quality of life and the overall prognosis [67]. Therefore, the treatment of cryoglobulinemic skin ulcers is particularly challenging in the clinical practice. These lesions are prevalent in the legs and feet, where other pathogenetic co-factors may be associated (venous insufficiency, arteriosclerosis), that are not rare in older patients with comorbidities and frequently treated with steroids [67]. Fig. (5) summarizes the therapeutic strategy for cryoglobulinemic skin ulcers, including both systemic (immunosuppressors, corticosteroids, and/or plasma exchange) and local treatments [67]. Among systemic treatments, the anti- CD20 monoclonal antibody rituximab represents one of the most effective and frequently employed therapy [55, 56, 59]; while, available data focusing on local therapeutic approach are generally limited to anecdotal observations [67]. Local treatments consisted of sharp or surgical debridement, as well as interactive dressing according to the condition of wound bed, perilesional skin, and the possible presence of infection, usually detected in the majority of patient with skin ulcers. Analgesic treatment is mandatory for background pain due to skin ulcers, as well as for procedural pain; this latter is critical for an effective local skin ulcers management. The majority of cryoglobulinemic skin ulcers heal at a variable time interval according to the severity of the single lesion; only a low percentage of patients with very severe, non-healing skin ulcers need amputation [67]. Overall, a trained team of operators with specific experience might be involved for an integrated therapeutic approach of these very challenging manifestations. 


\section{Therapeutic Strategies of Cryoglobulinemic Skin Ulcers (SU)}

\section{Systemic treatment Local treatment}

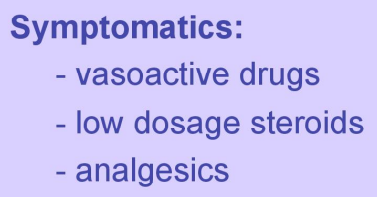

\section{Wound bed preparation: \\ - remove necrotic tissue \\ - prevent/treat infections \\ - moisture balance \\ - stimulate epithelial advancement \\ - procedural pain treatment}

\section{Growth factors:}

- platelet gel

\section{Autologous skin grafting}

Fig. (5). Therapeutic strategy of cryoglobulinemic ulcers should be based on both local and systemic therapies (see also text). After clinical work-up considering the entire MCs, including eventual comorbidities (venous insufficiency, arteriosclerosis, diabetes, ...), and careful examination of the ulcer characteristics, the systemic treatment may be based on etiological, pathogenetic, and/ or symptomatic therapies. More aggressive combined treatments with immunosuppressors, high dosage of steroids, and plasma aphaeresis may be necessary in the presence of very severe, non-healing skin ulcers. Long-term administration of analgesics is often necessary, also to achieve compliance during local treatment, which should be carried out at a wound care clinic. Wound bed preparation, which consider the prevention and the treatment of infections, is crucial for the healing of these skin ulcers.

\section{CONFLICT OF INTEREST}

The author(s) confirm that this article content has no conflicts of interest.

\section{ACKNOWLEDGEMENTS}

Declared none.

\section{REFERENCES}

[1] Meltzer M, Franklin EC, Elias K, McCluskey RT, Cooper N. Cryoglobulinemia. A clinical and laboratory study. II. Cryoglobulins with rheumatoid factor activity. Am J Med 1966; 40: 837-56.

[2] Ferri C. Mixed cryoglobulinemia. Orphanet J Rare Dis 2008; 3: 25.

[3] Brouet JC, Clouvel JP, Danon F, Klein M, Seligmann M: Biologic and-clinical significance of cryoglobulins. Am J Med 1974; 57:775-88.

[4] Zignego AL, Giannini C, Ferri C. Hepatitis C virus-related lymphoproliferative disorders: an overview. World J Gastroenterol 2007; 13:2467-78

[5] Ferri C, Sebastiani M, Saadoun D, Cacoub P. Cryoglobulinemia and hepatitis $\mathrm{C}$ virus. In, JWJ Bijsma, ed. EULAR compendium on rheumatic diseases, 2012. Chapter 42; London: BMJ Publishing Group Ltd. 2012; pp 1042-1071.

[6] Lenzi M, Johnson PJ, McFarlane IG, et al. Antibodies to hepatitis $\mathrm{C}$ virus in autoimmune liver disease: evidence for geographical heterogeneity. Lancet. 1991; 338: 277-80.

[7] Pascual M, Perrin L, Giostra E, Schifer JA. Hepatitis C virus in patients with Cryoglobulinemia type II. J Infect Dis 1990; 162: 569-70.

[8] Abel G, Zhang QX, Agnello V. Hepatitis C virus infection in type II mixed cryoglobulinemia (review). Arthritis Rheum 1993; $36: 1341-9$
[9] Ferri C, Greco F, Longombardo G, et al. Association between hepatitis $\mathrm{C}$ virus and mixed cryoglobulinemia. Clin Exp Rheumatol 1991; 9: 621-4

[10] Lunel F, Musset L, Cacoub P, et al. Cryoglobulinemia in chronic liver diseases: role of hepatitis $\mathrm{C}$ virus and liver damage. Gastroenterology 1994; 106: 1291-300

[11] Ferri C, Antonelli A, Mascia MT, et al. HCV-related autoimmune and neoplastic disorders: the HCV sindrome. Dig Liver Dis 2007 39 (Suppl. 1): S13-21.

[12] Monti G, Galli M, Invernizzi F, et al. Italian Group for the Study of Cryoglobulinaemias. Cryoglobulinaemias: a multi-centre study of the early clinical and laboratory manifestations of primary and secondary disease. QJM 1995; 88: 115-26.

[13] Ferri C, Sebastiani M, Giuggioli D, et al. Mixed cryoglobulinemia demographic, clinical, and serological features, and survival in 231 patients. Semin Arthritis Rheum 2004; 33: 355-74.

[14] Perez-Alamino R, Espinoza LR. Non-infectious cryoglobulinemia vasculitis (CryoVas): update on clinical and therapeutic approach Curr Rheumatol Rep. 2014; 16: 420.

[15] Zignego AL, Macchia D, Monti M, et al. Infection of periphera mononuclear blood cells by hepatitis C virus. J Hepatol 1992; 15: 382-6.

[16] Ferri C, Monti M, La Civita L, et al. Infection of peripheral blood mononuclear cells by hepatitis $\mathrm{C}$ virus in mixed cryoglobulinemia. Blood 1993; 82: 3701-4.

[17] Pileri P, Uematsu Y, Campagnoli S, et al. Binding of hepatitis C virus to CD81. Science 1998; 282: 938-41.

[18] De Re V, Caggiari L, Simula MP, et al. Role of the HLA class II: HCV-related disorders. Ann NY Acad Sci 2007; 1107: 308-18.

[19] Gorevic PD. Rheumatoid factor, complement, and mixed cryoglobulinemia. Clin Dev Immunol 2012; 2012: 439018.

[20] Dammacco F, Sansonno D, Piccoli C, Racanelli V, D'Amore FP, Lauletta $\mathrm{G}$. The lymphoid system in hepatitis $\mathrm{C}$ virus infection: autoimmunity, mixed cryoglobulinemia, and Overt B-cell malignancy. Semin Liver Dis 2000; 20: 143-57. 
[21] Zignego AL, Ferri C, Giannelli F, et al. Prevalence of BCL-2 rearrangement in hepatitis $\mathrm{C}$ virus-related mixed cryoglobulinemia with or without complicating B-cell lymphoma. Ann Int Med 2002; 137: 571-80.

[22] Zignego AL, Gragnani L, Piluso A, et al. Virus-driven autoimmunity and lymphoproliferation: the example of HCV infection. Expert Rev Clin Immunol 2015; 11:15-31.

[23] Ferri C, Pileri S, Zignego AL. In: Infectious causes of cancer: targets for intervention. Totowa, NJ; Goedert JJ, editor. The Humana Press Inc 2000; 349-68.

[24] Peveling-Oberhag J, Arcaini L, Hansmann ML, Zeuzem S. Hepatitis C-associated B-cell non-Hodgkin lymphomas. Epidemiology, molecular signature and clinical management. J Hepatol 2013; 59: 169-77.

[25] Forghieri F, Luppi M, Barozzi P, et al. Pathogenetic mechanisms of hepatitis $\mathrm{C}$ virus-induced B-cell lymphomagenesis. Clin Dev Immunol 2012; 2012:807351.

[26] Ferri C, Sebastiani M, Giuggioli D, et al. Hepatitis C virus syndrome:A constellation of organ-and non-organ specific autoimmune disorders, B-cell non-Hodgkin's lymphoma, and cancer. World J Hepatol 2015; 7 : 327-43.

[27] De Vita S, Soldano F, Isola M, et al. Preliminary classification criteria for the cryoglobulinaemic vasculitis. Ann Rheum Dis 2011; 70:1183-1190.

[28] Buskila D. Hepatitis C-associated arthritis. Curr Opin Rheumatol 2000; 12: 295-9.

[29] Fadda P, La Civita L, Zignego AL, Ferri C. Hepatitis C virus infection and arthritis. A clinico-serological investigation of arthritis in patients with or without cryoglobulinemic syndrome. Reumatismo 2002; 54: 316-23.

[30] Ferri C, La Civita L, Cirafisi C, et al. Peripheral neuropathy in mixed cryoglobulinemia: clinical and electrophysiologic investigations. J Rheumatol 1992; 19:889-95.

[31] Saadoun D, Bieche I, Maisonobe T, et al. Involvement of chemokines and type 1 cytokines in the pathogenesis of hepatitis $\mathrm{C}$ virusassociated mixed cryoglobulinemia vasculitis neuropathy. Arthritis Rheum 2005; 52:2917-25.

[32] Boonyapisit K, Katirji B. Severe exacerbation of hepatitis Cassociated vasculitic neuropathy following treatment with interferon alpha: a case report and literature review. Muscle Nerve 2002; 25: 909-13.

[33] Colombo M. Hepatitis C virus and hepatocellular carcinoma. Baillieres Best Practice \& Research Clinical Gastroenterology. 1999; 13: 519-28.

[34] Vitali C. Immunopathologic differences of Sjögren's syndrome versus sicca syndrome in $\mathrm{HCV}$ and HIV infection. Arthritis Res Ther 2011; 13: 233.

[35] Ramos-Casals M, Loustaud-Ratti V, De Vita S, et al. SS-HCV Study Group. Sjogren syndrome associated with hepatitis $\mathrm{C}$ virus: a multicenter analysis of 137 cases. Medicine 2005; 84: 81-9.

[36] Roccatello D, Fornasieri A, Giachino O, et al. Multicenter study on hepatitis $\mathrm{C}$ virus-related cryoglobulinemic glomerulonephritis. Am J Kidney Dis 2007; 49: 69-82.

[37] Uchiyama-Tanaka Y, Mori Y, Kishimoto N, et al. Membranous glomerulonephritis associated with hepatitis $\mathrm{C}$ virus infection: case report and literature review. Clin Nephrol 2004; 61: 14450.

[38] Zuo KJ, Ménard HA, Amre R, Colmegna I. Mixed cryoglobulinemia-associated vasculitis. Arthritis Rheumatol 2014; 66: 443.

[39] Aliannejad R, Ghanei M. Hepatitis C and pulmonary fibrosis. Hepat Mon 2011; 11:71-3.

[40] Antonelli A, Ferri C, Ferrari SM, Colaci M, Fallahi P. Immunopathogenesis of HCV-related endocrine manifestations in chronic hepatitis and mixed cryoglobuliemia. Autoimmun Rev 2008; 8: 1823.

[41] Fallahi P, Ferrari SM, Giuggioli D, et al. Thyroid inolvement in hepatitis C-associated mixed cryoglobulinemia. Hormones 2014; 13:16-23.

[42] Antonelli A, Ferrari SM, Corrado A, Di Domenicantonio A, Fallahi P. Autoimmune thyroid disorders. Autoimmun Rev 2015; 14: 17480 .
[43] Antonelli A, Ferrari SM, Giuggioli D, et al. Hepatitis C virus infection and type 1 and type 2 diabetes mellitus.World J Diabetes. 2014; 5: 586-600.

[44] Zuckerman E, Keren D, Slobodin G, et al. Treatment of refractory, symptomatic, hepatitis $\mathrm{C}$ virus related mixed cryoglobulinemia with ribavirin and interferon-alpha. J Rheumatol 2000; 27: 2172-8.

[45] Mazzaro C, Zora F, Caizzi M, et al. Treatment with peg interferon alfa-2b and ribavirin of hepatitis $\mathrm{C}$ virus-associated mixed cryoglobulinemia: a pilot study. J Hepatol 2005; 42: 632-8.

[46] Ferri C, Sebastiani M, Antonelli A, Colaci M, Manfredi A, Giuggioli D. Current treatment of hepatitis C-associated rheumatic diseases. Arthritis Res Ther 2012; 14: 215.

[47] Saadoun D, Resche-Rigon M, Thibault V, Piette JC, Cacoub P. Antiviral therapy for hepatitis $\mathrm{C}$ virus--associated mixed cryoglobulinemia vasculitis: a long-term followup study. Arthritis Rheum 2006; 54: 3696-706.

[48] Lidove O, Cacoub P, Hausfater P, et al. Cryoglobulinemia and hepatitis $\mathrm{C}$ : worsening of peripheral neuropathyafter interferon alpha treatment. Gastroenterol Clin Biol 1999; 23: 403-6.

[49] Cacoub P, Terrier B, Saadoun D. Hepatits C virus-induces vascultis:therapeutic options. Ann Rheum Dis 2014; 73: 24-30.

[50] Gragnani L, Fognani E, Piluso A, et al. MaSVE Study Group. Long-term effect of HCV eradication in patients with mixed cryoglobulinemia: a prospective, controlled, open-label, cohort study. Hepatology 2015; 61: 1145-53.

[51] Saadoun D, Resche Rigon M, Pol S, et al. PegIFN/ribavirin/protease inhibitor combination in sevre hepatitis $\mathrm{C}$ virus-associasted mixed cryoglobulinemia vasculits. J Hepatol 2015; 62: 24-30.

[52] Cornella SL, Stine JG, Kelly V, Caldewell SH, Shah Nl. Persistence of mixed cryoglobulinemia despite cure of hepatitis $\mathrm{C}$ with new oral antiviral therapy including direct-acting antiviral sofosbuvir: A case series. Postgrad Med 2015; 127(4): 413-7.

[53] Garcia A, Fernandez S, Toro F, De Sanctis JB. An overview of hepatitis C vaccines. Recent Pat Inflam Allergy Drug Discov 2014; 8: 85-91.

[54] Pietrogrande M, De Vita S, Zignego AL, et al. Recommendations for the management of mixed cryoglobulinemia syndrome in hepatitis C virus-infected patients. Autoimmun Rev 2011; 10: 444-54.

[55] Ferri C, Cacoub P, Mazzaro C, et al. Treatment with rituximab in patients with mixed cryoglobulinemia syndrome: Results of multicenter cohort study and review of the literature. Autoimmun Rev 2011; 11: 48-55.

[56] Dammacco F, Tucci FA, Lauletta G, et al. Pegylated interferonalpha, ribavirin, and rituximab combined therapy of hepatitis $\mathrm{C}$ virus-related mixed cryoglobulinemia: a long-term study. Blood 2010; 116: 343-53.

[57] Stefanutti C, Di Giacomo S, Mareri M, et al. Immunoadsorption apheresis (Selesorb) in the treatment of chronic hepatitis $\mathrm{C}$ virusrelated type 2 mixed cryoglobulinemia. Transfus Apher Sci 2003; 28: 207-214.

[58] Ferri C, Pietrogrande M, Cecchetti C, et al. Low-antigen-content diet in the treatment of mixed cryoglobulinemia patients. Am J Med 1989; 87: 519-24.

[59] De Vita S, Quartuccio L, Isola M, et al. A randomized controlled trial of rituximab for the treatment of severe cryoglobulinemic vasculitis. Arthritis Rheum 2012; 64: 843-53.

[60] Sansonno D, De Re V, Lauletta G, Tucci FA, Boiocchi M, Dammacco F. Monoclonal antibody treatment of mixed cryoglobulinemia resistant to interferon alpha with an anti-CD20. Blood 2003; 101: 3818-26.

[61] Zaja F, De Vita S, Mazzaro C, et al. Efficacy and safety of rituximab in type II mixed cryoglobulinemia. Blood 2003; 101: 3827-34.

[62] Antonelli A, Ferri C, Fallahi P, et al. Alpha-chemokine CXCL10 and beta-chemokine CCL2 serum levels in patients with hepatitis $\mathrm{C}$-associated cryoglobulinemia in the presence or absence of autoimmune thyroiditis. Metabolism 2008; 57: 1270-7.

[63] Antonelli A, Fallahi P, Ferrari SM, et al. Serum Th1 (CXCL10) and Th2 (CCL2) chemokine levels in children with newly diagnosed Type 1 diabetes: a longitudinal study. Diabet Med 2008; 25 : $1349-53$. 
[64] Antonelli A, Ferri C, Fallahi P, et al. Clinical and subclinical autoimmune thyroid disorders in systemic sclerosis. Eur J Endocrinol 2007; 156: 431-7.

[65] Antonelli A, Ferri C, Fallahi P, et al. Thyroid disorders in chronic hepatitis $C$ virus infection. Thyroid 2006; 16: 563-72.

[66] Antonelli A, Ferri C, Fallahi P, et al. Hepatitis C virus infection: evidence for an association with type 2 diabetes. Diabetes Care 2005; $28: 2548-50$.
[67] Giuggioli D, Manfredi A, Lumetti F, Sebastiani M, Ferri C. Cryoglobulinemic vasculitis and skin ulcers. Our therapeutic strategy and review of the literature. Semin Arthritis Rheum 2015; 44: 51826. 